\title{
Synergistic effects of overexpression of BMP-2 and TGF- $\beta 3$ on osteogenic differentiation of bone marrow mesenchymal stem cells
}

\author{
YILIN WANG ${ }^{1 *}$, TIAN HE ${ }^{2 *}$, JIE LIU ${ }^{1}$, HONGZHI LIU ${ }^{2}$, \\ LUGANG ZHOU $^{2}$, WEI HAO ${ }^{2}$, YUJIE SUN ${ }^{2}$ and XIN WANG ${ }^{2}$
}

Departments of ${ }^{1}$ Biochip Laboratory, and ${ }^{2}$ Orthopedic Surgery, Yantai Yuhuangding Hospital Affiliated to Medical College of Qingdao University, Yantai, Shandong 264400, P.R. China

Received October 3, 2015; Accepted August 22, 2016

DOI: $10.3892 / \mathrm{mmr} .2016 .5961$

\begin{abstract}
Bone morphogenetic protein 2 (BMP-2) and transforming growth factor $\beta$ (TGF- $\beta$ ) isoforms are important in advancing bone regeneration. The aim of the present study was to investigate the positive and reciprocal effect of TGF- $\beta 3$, one of the three TGF- $\beta$ isoforms, on BMP-2 in promoting osteogenic differentiation. Exogenous BMP- 2 and TGF- $\beta 3$ genes were separately, and in combination, overexpressed in rabbit bone marrow-derived mesenchymal stem cells (rBMSCs). Expression levels of BMP- 2 and TGF- $\beta 3$ were evaluated using reverse-transcription-polymerase chain reaction (RT-PCR) and Western blotting assays. Furthermore, the osteogenic differentiation capacities of BMSCs were assessed by measuring Alizarin Red S staining, an alkaline phosphatase activity assay, and quantification of the osteogenic-specific genes, Runt-related transcription factor 2 (Runx2) and Osterix (Osx). Using lentiviral-mediated transfection, robust co-transfection efficiency of $>90 \%$ was achieved. RT-PCR and immunoblotting results indicated a marked elevated expression of BMP-2 and TGF- $\beta 3$ in rBMSCs undergoing co-transfection, compared with transfection with BMP-2 or TGF- $\beta 3$ alone, indicating that BMP- 2 and TGF- $\beta 3$ are synergistically expressed in rBMSCs. Furthermore, enhanced osteogenic differentiation was observed in rBMSCs co-transfected with BMP-2/TGF- $\beta 3$. The present study successfully delivered BMP-2 together with TGF- $\beta 3$ into rBMSCs with high efficiency for the first time. Furthermore, TGF- $\beta 3$ overexpression was demonstrated to enhance the osteogenic efficacy of BMP-2 in rBMSCs,
\end{abstract}

Correspondence to: Dr Tian He or Dr Xin Wang, Department of Orthopedic Surgery, Yantai Yuhuangding Hospital Affiliated to Medical College of Qingdao University, 20 East Yuhuangding Road, Zhifu, Yantai, Shandong 264400, P.R. China

E-mail: hunterhe@hotmail.com

E-mail: wangxin2055@hotmail.com

*Contributed equally

Key words: bone morphogenic protein-2, transforming growth factor- $\beta 3$, osteogenesis, rabbit bone marrow-derived mesenchymal stem cells, osteogenic differentiation, synergistic effects and vice versa. This provides a potential clinical therapeutic approach for regenerating the function of osseous tissue, and may present a promising strategy for bone defect healing.

\section{Introduction}

Application of nucleic acid recombination technology, particularly when conducted with stem cells, has advanced gene therapy from bench to bedside $(1,2)$. Gene therapy is used in correcting inherited disorders, but also in healing diverse acquired diseases, such as carcinoma, heart failure, neurodegenerative and metabolic disorders and acquired immune deficiency syndrome (3-8). An increasing number of clinical trials have revealed that gene transplantation- and stem cell-based bone tissue engineering are effective therapeutic options for promoting osteogenesis in bone and joint surgery (9-11).

Typically, bone regeneration is a complicated process that involves a series of cellular signaling pathways that are triggered or regulated by multiple growth factors and biomolecules. The transforming growth factor- $\beta$ (TGF- $\beta$ ) superfamily comprises a group of multifunctional peptide growth factors exerting a marked impact on the osteogenic potential of progenitor cells. Initiation of canonical TGF- $\beta /$ Smad signaling leads to expression of osteogenic genes, which is followed by osteogenic differentiation of various stem cells (12-14). Among TGF- $\beta$ growth factors, the subfamily of bone morphogenetic proteins (BMPs) has been extensively studied and a number of mediators in cartilage and bone formation have been identified (15). As a member of the BMP family, BMP-2 is important in the bone remodeling process $(16,17)$. It was demonstrated that BMP-2 had a role in bone defect healing when delivered with a carrier substance $(15,18)$ and was able to induce bone synthesis within two weeks following implantation of transfected cells (19). In addition, evidence has shown that a short period of BMP-2 expression is sufficient to induce bone regeneration (20), hence the hypothesis that BMP-2 is one of the most active promoters for differentiation of mesenchymal cells to osteoblasts in vitro, in addition to being able to induce bone formation in vivo (21).

TGF- $\beta 3$, one of the three TGF- $\beta$ isoforms, was generally recognized to facilitate chondrogenic differentiation of precursor cells $(22,23)$, however, a previous study has also 
shown it may have a dose-dependent inhibitory effect on osteogenesis (24). By contrast, previous studies have also demonstrated that mammalian TGF- $\beta 3$ is a regulator implicated in the early stages of osteoblastic differentiation (25-27). Klar et al (28) observed that TGF- $\beta 3$ signaling elicited endochondral bone differentiation by regulating BMP activity, and, thus, induction of bone formation. Furthermore, the previous study reported that TGF- $\beta 3$ stimulates bone synthesis via upregulation of endogenous BMP-2. Therefore, the role of TGF- $\beta 3$ in bone formation is of considerable interest and remains to be elucidated.

In a previous study, co-delivery of BMP- 2 and TGF- $\beta 3$ was demonstrated to be more effective than single gene-transfection in promoting ossification of the annulus fibrosus (29). Therefore, the present study simultaneously expressed BMP-2 and TGF- $\beta 3$ genes in rBMSCs and determined their expression status in vitro so as to elucidate whether they can be synergistically expressed in vivo. Further investigation into the effect on bone differentiation and regeneration following delivery was also conducted to elucidate possible underlying mechanisms of their synergistic effect.

\section{Materials and methods}

Experimental animals. A total of two male and two female New Zealand white rabbits (weight, 400-500 g; age, 4 weeks) were obtained from the Animal Experimentation Center of Qingdao University (Qingdao, China). Rabbits were housed individually in standard cages and maintained under standard laboratory conditions (relative humidity, $50 \pm 10 \%$; temperature $25 \pm 1^{\circ} \mathrm{C} ; 12$-h light/dark cycles), with access to food twice a day and free access to water. Rabbits were sacrificed by peritoneal injection with $10 \mathrm{ml} / \mathrm{kg}$ of $10 \%$ chloral hydrate.

All experiments were conducted in accordance with the Guidance Suggestions for the Care and Use of Laboratory Animals of the Ministry of Science and Technology of China (30). Animal procedures were approved by the Medical Ethics Committee of Yantai Yuhuangding Hospital (Yantai, China).

Isolation and culture of rBMSCs. BMSCs were isolated from the tibial and femoral shafts of the rabbits. The ends of the femora were cut off at the epiphysis, and the marrow was flushed out using $20 \mathrm{ml} \alpha$-minimum essential medium ( $\alpha$-MEM; Hyclone; GE Healthcare Life Sciences, Logan, UT, USA) supplemented with $10 \%$ fetal bovine serum (FBS; Gibco; Thermo Fisher Scientific, Inc., Waltham, MA, USA), 100 U/ml of penicillin and $100 \mu \mathrm{g} / \mathrm{ml}$ of streptomycin (Sigma-Aldrich; Merck Millipore, Darmstadt, Germany) with a 20-gauge needle. The collected cells were collected into $25-\mathrm{cm}^{2}$ cell culture flasks (Nalge Nunc International, Penfield, NY, USA) containing $5 \mathrm{ml}$ of the aforementioned medium. The medium was changed after $48 \mathrm{~h}$ to remove non-adherent cells and then renewed every day. Cultures were maintained in a humidified atmosphere of $5 \% \mathrm{CO}_{2}$ at $37^{\circ} \mathrm{C}$. Following reaching $70-80 \%$ confluence (after $\sim 1$ week), the cells were harvested using $0.25 \%$ trypsin (Hyclone; GE Healthcare Life Sciences) and cell concentration was adjusted to $1 \times 10^{6}$ cells $/ 1$. Following passage, the cells were plated in flasks and cultured until third-passage rBMSCs (P3) were obtained.
Plasmid construction and transfection of lentivirus vectors. The DNA fragment that encoded human BMP-2 or TGF- $\beta 3$ that had been cloned into the pIRES vector, was provided by the Central Laboratory at the Medical School of Qingdao University (Qingdao, China). Corresponding lentivirus packaging plasmids were produced by Shanghai GenePharma Co., Ltd. (Shanghai, China).

P3 rBMSCs were divided into four groups as follows: i) Group I, negative controls, consisting of untransfected rBMSCs or rBMSCs transfected with an empty vector (vehicle); ii) group II, rBMSCs transfected with lentivirus carrying green fluorescent protein (GFP)/BMP-2; iii) group III, rBMSCs transfected with lentivirus carrying TGF- $\beta 3$; iv) group IV, rBMSCs co-transfected with BMP-2 and TGF- $\beta 3$. The procedure of transfection was performed as previously described (30). Briefly, rBMSCs were seeded in 6-well culture plates and sequentially infected with lentivirus (Shanghai GenePharma Co., Ltd.) encompassing the indicated genes [multiplicity of infection (MOI) $=20,30,40,50,55$ and 60] or negative short hairpin RNA (Lenti-shcontrol) at $80 \%$ confluency $(\sim 500,000$ cells/well) using Polybrene $(8 \mu \mathrm{g} / \mathrm{ml}$ culture medium; Sigma-Aldrich; Merck Millipore). The efficiency of transfection was estimated by detecting the proportion of GFP-positive rBMSCs under a fluorescence microscope.

Reverse transcription-polymerase chain reaction (RT-PCR). Following transfection with corresponding plasmids, after one week, total RNA was isolated from cells using TRIzol (Takara Bio, Inc., Otsu, Japan) according to the manufacturer's protocols, and subsequently digested with RNase-free DNase I. The concentration and quality of extracted RNA was evaluated by calculating the absorbance at $260 \mathrm{~nm}\left(\mathrm{~A}_{260}\right)$ and the $\mathrm{A}_{260 / 280}$ ratio, respectively, using a spectrophotometer. cDNA was generated by reverse transcription using $1 \mu \mathrm{g}$ RNA as a template, and RT-PCR was subsequently conducted. To evaluate BMP-2 and TGF- $\beta 3$ expression, quantities of target genes were normalized to that of the housekeeping gene GAPDH, which served as the internal control. The sequences of forward and reverse primers (synthesized at Sangon Biotech Co., Ltd., Shanghai, China) used in the present study were as follows: Forward, 5'-CCAACCATGGATTCGTGGTG-3' and reverse, 5'-GGT ACAGCATCGAGATAGCA-3' for BMP-2; forward, 5'-TGG CTGTTGAGAAGAGAGTCC-3' and reverse, 5'-TGCTTC AGGGTTCAGAGTGTT-3' for TGF- $\beta 3$; and forward, 5'-GCC TGGAGAAAGCTGCTAAGTA-3' and reverse, 5'-CGTTGT CATACCAGGAAATGAG-3' for GAPDH. The amplification profile was $95^{\circ} \mathrm{C}$ for $5 \mathrm{~min}$, followed by 38 cycles $(36$ cycles for GAPDH) of denaturation at $98^{\circ} \mathrm{C}$ for $10 \mathrm{sec}$, hybridization annealing at $62^{\circ} \mathrm{C}\left(60^{\circ} \mathrm{C}\right.$ for GAPDH $)$ for $30 \mathrm{sec}$, and extension at $72^{\circ} \mathrm{C}$ for $45 \mathrm{sec}$, followed by an extension cycle for $10 \mathrm{~min}$ at $72^{\circ} \mathrm{C}$. PCR products were visualized on $1.0 \%$ (w/v) agarose gels stained with ethidium bromide. The band densities were quantified by detecting absorbance values and analyzed using Quantity One software (version 4.6; Bio-Rad Laboratories, Inc., Hercules, CA, USA) to measure mRNA levels. The signals were normalized to GAPDH expression. Experiments were performed in triplicate.

Osteogenic induction. To induce osteogenic differentiation, the rBMSCs were cultured in $\alpha$-MEM 
containing $10 \%$ FBS, $1 \%$ penicillin and streptomycin, $50 \mu \mathrm{g} / \mathrm{ml}$ ascorbic acid (Sigma-Aldrich; Merck Millipore), 10 mM $\beta$-glycerophosphate (Sigma-ldrich; Merck Millipore), and $10 \mathrm{nM}$ dexamethasone (Sigma Aldrich; Merck Millipore). The culture medium was exchanged every 3 days.

Alizarin Red S staining. Staining was performed as described in our previous study (30). Briefly, on day 21, cells were fixed and stained with Alizarin Red S staining solution. The stained monolayers were then washed 3 times with phosphate-buffered saline (PBS) and visualized using phase microscopy with an inverted microscope (DMI4000B, Leica Microsystems GmbH, Wetzlar, Germany).

Alkaline phosphatase (ALP) activity. The activity of ALP and total protein quantity were assessed on days $0,3,7,14$ and 21 . The lysates was determined by LabAssay ALP colorimetric assay kit (Wako Pure Chemical Industries, Ltd., Osaka, Japan). Total proteins were determined by BCA Protein assay kit (Beyotime Institute of Biotechnology, Haimen, China) following the standard protocol. The activity of ALP was calculated as phosphorylated nitrophenol release in $\mathrm{n} / \mathrm{mol} / \mathrm{h}$ and was further normalized to the cell protein input. Each sample was assessed in triplicate.

Protein extraction and Western blotting analysis. Cultures were washed three times with PBS, and sequentially harvested cells were pelleted by centrifugation at $8,000 \times \mathrm{g}$ for $15 \mathrm{~min}$ at room temperature. Cell pellets were then resuspended in radioimmunoprecipitation assay lysis buffer containing $1 \%$ phenylmethylsulfonyl fluoride protease inhibitor, before samples were incubated on ice for $1 \mathrm{~h}$. Lysates were subjected to ultrasonication on ice for further lysing and cell debris was removed by centrifugation at $16,000 \times \mathrm{g}$ for $10 \mathrm{~min}$ at $4^{\circ} \mathrm{C}$. Following centrifugation, protein concentration was determined using a QuantiPro BCA assay kit (Sigma-Aldrich; Merck Millipore) and the protein supernatant was kept at $-80^{\circ} \mathrm{C}$ for future analysis

For immunoblotting, proteins $(\sim 40 \mu \mathrm{g})$ were separated on $8 \%$ SDS-PAGE and transferred to polyvinylidene difluoride membranes at $60 \mathrm{~V}$ for $1 \mathrm{~h}$ at $4^{\circ} \mathrm{C}$. The membranes were blocked with milk and then incubated overnight at $4^{\circ} \mathrm{C}$ with primary antibodies against mouse monoclonal BMP-2 (dilution, 1:1,000; Abcam, Cambridge, MA, USA; cat no. ab6285), rabbit polyclonal TGF- $\beta 3$ (dilution, 1:1,000; Abcam; cat no. ab15537), Runx2-C-terminal region (dilution, 1:1,000; Aviva Systems Biology Corporation, San Diego, CA, USA; cat no. ARP38453_P050), Osx (dilution, 1:200; Beijing Biosynthesis Biotechnology Co., Ltd., Beijing, China; cat no. bs-1110R), or mouse monoclonal $\beta$-actin (1:2,000; Abcam; cat no. ab6276,), followed by rinsing 3 times with PBS with Tween 20 for $30 \mathrm{~min}$, and subsequently incubated with secondary antibodies at room temperature for $1 \mathrm{~h}$. Secondary antibodies were horseradish peroxidase (HRP)-conjugated goat anti-mouse IgG (dilution, 1:2,000; Sigma-Aldrich; Merck Millipore; cat no. A0168) or HRP-conjugated goat anti-rabbit IgG (dilution, 1:3,000; Sigma-Aldrich; Merck Millipore; cat no. A0545). The membrane was washed with PBS containing 0.05\% Tween 20 three times, for $10 \mathrm{~min}$ each time, prior to being developed using the Immobilon ${ }^{\mathrm{TM}}$ Western Chemiluminescent HRP Substrate (Merck Millipore; cat. no. WBKLS0500).
Statistical analysis. Data are presented as the mean \pm standard deviation. Significance between various treatment samples was calculated using the Student's t-test. All statistical analyses were conducted with SPSS 19.0 software (IBM SPSS, Armonk, NY, USA). $\mathrm{P}<0.05$ was considered to indicate a statistically significant difference.

\section{Results}

Marked high transfection efficiency following co-transfection. To evaluate the efficiency of lentivirus-mediated transfection, expression of a vector encoding GFP in rBMSCs was visualized using fluorescence microscopy. GFP was expressed in rBMSCs with high intensity and lasted stably, gradually reaching a peak value at $72 \mathrm{~h}$ after transfection. Images from three random fields were captured for each well and GFP-positive cells per microscope field were counted. The ratio of GFP-positive cells compared with total cells was defined as the transfection efficiency. As presented in Fig. 1A, a robust transfection efficiency of $>90 \%$ was observed in each experimental group when cells were transfected with lentiviral-mediated BMP-2, TGF- $\beta 3$, or BMP-2/TGF- $\beta 3$ genes at an MOI of 40, 40 and 55, respectively. Furthermore, the incidence of suspended cells increased in the group undergoing co-transfection compared with single-gene transfected counterparts, indicating decreased proliferation of rBMSCs when co-transfected. However, it did not affect the transfection rate. This demonstrated that the present study efficiently delivered BMP- 2 and TGF- $\beta 3$ into rBMSCs together, which has been technically challenging to this point.

BMP-2 and TGF- $\beta 3$ were mutually upregulated in co-transfected rBMSCs. The expression status of corresponding exogenous genes was detected based on RT-PCR analysis, 5 days after transfection. The results indicated that expression levels of BMP-2 and TGF- $\beta 3$ in lentivirus-treated rBMSCs were markedly increased compared with those of untreated cells. Notably, expression of BMP-2 in rBMSCs was significantly increased when co-expressed with TGF- $\beta 3$, in comparison to that in rBMSCs transfected with BMP-2 $(\mathrm{P}=0.019)$. Similarly, increased TGF- $\beta 3$ mRNA level was exacerbated by BMP-2 $(\mathrm{P}=0.021$; Fig. 1B), indicating that BMP-2 and TGF- $\beta 3$ were expressed in rBMSCs when cultured in vitro.

This RT-PCR result was consistent with results obtained by assessing BMP- 2 and TGF- $\beta 3$ protein expression levels using Western blotting. It was demonstrated that BMP- 2 or TGF- $\beta 3$ transfection significantly facilitated upregulation of the corresponding gene, and higher expression levels of BMP-2 and TGF- $\beta 3$ proteins were observed in co-transfected rBMSCs than in cells transfected with a single gene (Fig. 1C), consistent with the PCR results. The data collectively suggested that BMP-2 and TGF- $\beta 3$ synergistically induced expression of the other, with a possible association indicated by the mutual role they play in the bone-forming process. Following this, the present study next assessed the alteration of osteogenesis in post-co-expressed rBMSCs.

TGF- $\beta 3$ enhanced osteogenic function of BMP2. To further investigate the osteogenic function of rBMSCs undergoing BMP-2 and/or TGF- $\beta 3$ delivery, expression levels of Runx2, and Osx, the representative early osteogenic-specific markers, 

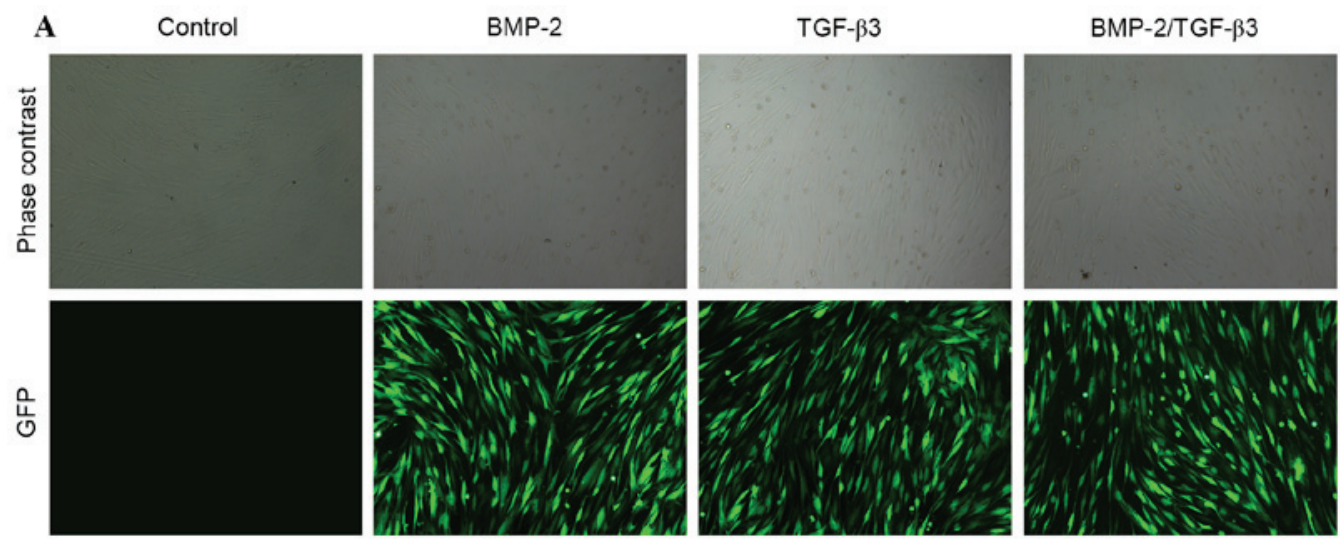

B
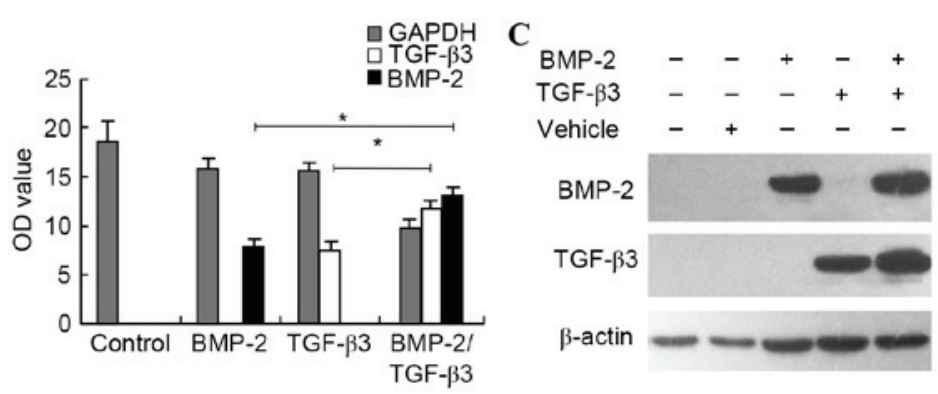

Figure 1. BMP-2 and TGF- $\beta 3$ were synergistically expressed in rBMSCs. (A) Strong green fluorescence intensity indicated a robust lentivirus-mediated transfection efficiency of rBMSCs (magnification, x100). Increased suspended cells in the co-transfection group indicated decreased proliferation of rBMSCs. The expression levels of BMP- 2 and TGF- $\beta 3$ were increased in the rBMSCs co-transfected with BMP-2/TGF- $\beta 3$ lentiviral particles, as assessed by (B) reverse transcription-polymerase chain reaction and $(\mathrm{C})$ western blotting. Data are presented as the mean \pm standard deviation from triplicate repeats. ${ }^{*} \mathrm{P}<0.05 \mathrm{vs}$. indicated groups. BMP-2, bone morphogenetic protein 2 ; TGF- $\beta 3$, transforming growth factor $\beta 3$; rBMSCs, rabbit bone marrow-derived mesenchymal stem cells; GFP, green fluorescent protein.

were estimated. As expected, BMP-2 and TGF- $\beta 3$ overexpression markedly upregulated Runx-2 and Osx expression levels (Fig. 2A). Notably, rBMSCs demonstrated increased expression of Runx 2 and Osx when co-transfected with BMP-2 and TGF- $\beta 3$, compared with those transfected with BMP-2 alone, which indicated that TGF- $\beta 3$ enhanced osteogenic differentiation capacity for BMP-2 in rBMSCs.

Following lentivirus infection, ALP activities were measured to examine the mechanism by which BMP-2 and/or TGF- $\beta 3$ overexpression affects the osteogenic differentiation process. Compared with the negative control, ALP activities in rBMSCs transfected with BMP- 2 and TGF- $\beta 3$ gradually increased with time. As presented in Fig. 2B, ALP activity in the BMP-2-transfected rBMSCs was higher than that in TGF- $\beta 3$ transfected stem cells $(\mathrm{P}=0.0353$ and $\mathrm{P}=0.023$ at days 3 and 7, respectively; $\mathrm{P}<0.01$ at days 14 and 21) possibly due to a more robust osteogenic activity of BMP-2. However, when co-transfected with BMP- 2 and TGF- $\beta 3$, rBMSCs presented significantly increased ALP activities at all time points compared with rBMSCs transfected with BMP2. $(\mathrm{P}=0.0187$ at day $3 ; \mathrm{P}<0.01$ at days 7, 14 and 21 ). The capacity of TGF- $\beta 3$ in osteogenic differentiation may be elevated by BMP-2, and BMP-2 mediated ossification was in turn enhanced by TGF- $\beta 3$ delivery.

In addition, osteogenic capabilities were characterized by examining the mineralization of the extracellular matrix using Alizarin Red S staining after 21 days of culture. As hypothesized, marginal mineralized nodules were observed in negative control groups with or without osteogenic introduction
(Fig. 2C). However, in agreement with data from the Western blotting, although there was no marked difference in the density of mineralized nodule areas between BMP- 2 and TGF- $\beta 3$ overexpressed rBMSCs, the proportion of mineralized nodules was notably increased in rBMSCs incubated with the BMP-2 and TGF- $\beta 3$ encapsulated lentivirus.

The results of the present study demonstrated that when acting together, BMP- 2 and TGF- $\beta 3$ increased promotion of osteogenic differentiation compared with when functioning individually.

\section{Discussion}

Stem cells have been extensively introduced to the field of clinical bioengineering, resulting from their ability to self-renew and differentiate into multiple types of cell. Research focus has shifted to the application of mesenchymal stem cells (MSCs) for therapeutic models primarily as MSCs may be favorably isolated from bone marrow aspiration and expand $>20$ population doublings without a loss of their potency of differentiation, with no untoward reaction in allogeneic MSC transplantation $(31,32)$. BMSCs are particularly promising in orthopedic surgery due to their osteoinductive potential $(33,34)$. Notably, osteogenic differentiation of BMSCs, coupled with maintenance of cell phenotypes following differentiation, requires induction of multiple growth factors and specific microenvironments $(21,35,36)$.

Transforming growth factors are known to be associated with the coordination of diverse physiological processes, 
A

B
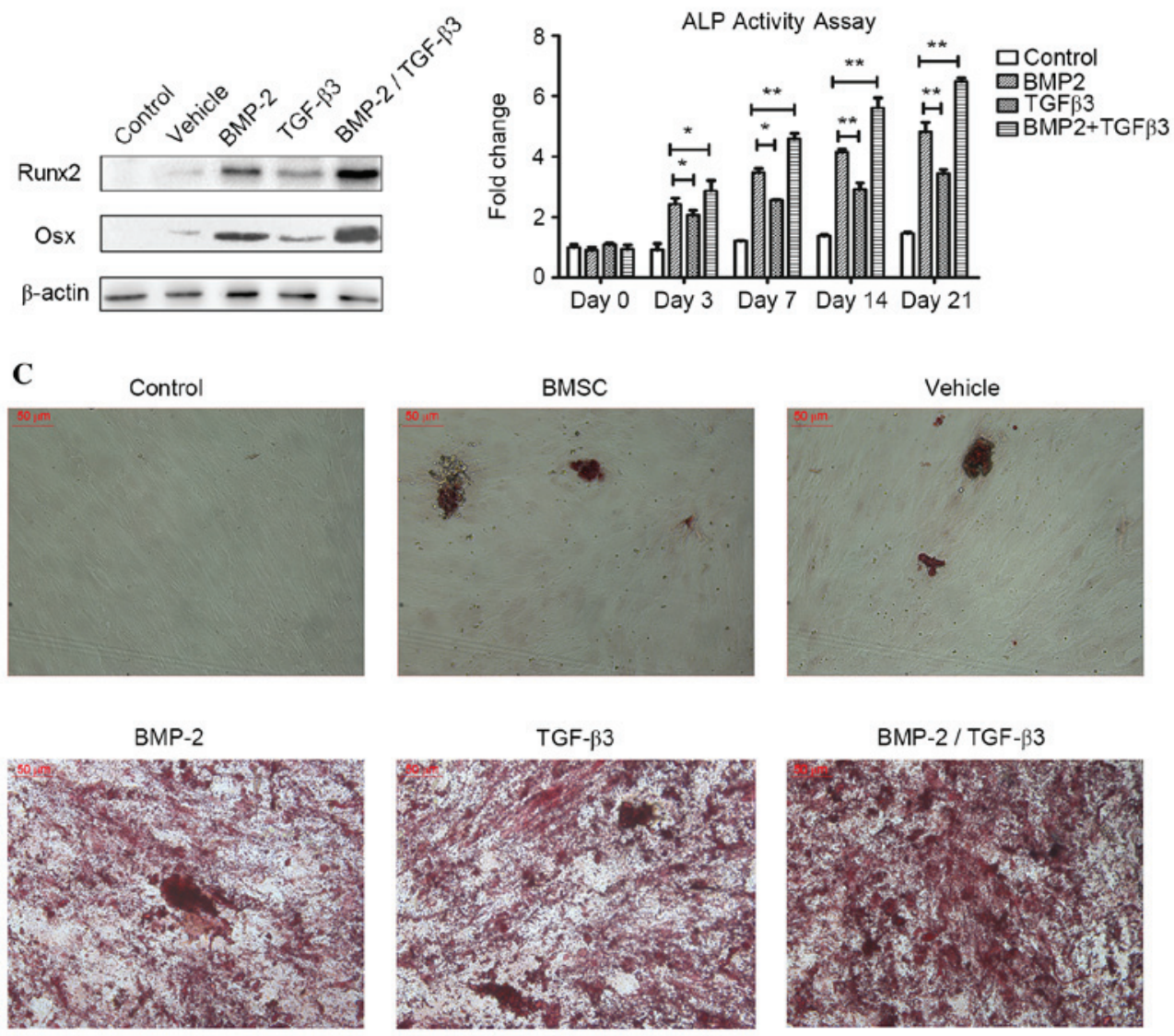

Figure 2. Mutual effect of BMP-2 and TGF- $\beta 3$ on osteogenic differentiation. (A) Increased expression levels of Runx 2 and Osx, which are early markers for osteogenic differentiation in osteogenic cultures, were observed in cells transfected with BMP-2 and TGF- $\beta 3$. (B) ALP activity of rabbit BMSCs osteogenic cultures was analyzed at the indicated time points. Data are presented as the mean \pm standard deviation. ${ }^{*} \mathrm{P}<0.05$ and ${ }^{* * *} \mathrm{P}<0.01$ vs. indicated groups. (C) Alizarin Red S staining was performed to visualize mineral deposition at day 21 post-transfection (magnification x 200). Marginal mineralized nodules were observed in negative control groups, however a markedly higher density of nodules were detected in BMSCs undergoing gene transfection, particularly in co-delivered cells. Control cells were stem cells normally cultured without osteogenesis induction treatment, while BMSC indicates cells undergoing induction but without exogenous gene transfection. BMP-2, bone morphogenetic protein 2 ; TGF- $\beta 3$, transforming growth factor $\beta$; Runx-2, Runt-related transcription factor 2; Osx, Osterix; ALP, alkaline phosphatase; BMSCs, bone marrow-derived mesenchymal stem cells.

including cellular proliferation and differentiation, embryogenesis, the immune response, and wound healing $(37,38)$. The TGF- $\beta$ superfamily principally comprises the TGF- $\beta$ subfamily (with three isoforms) (39), and the decapentaplegic Vg-related subfamily including BMPs. Numerous previous studies have demonstrated the osteogenic importance of BMP-2, a growth factor that belongs to the BMP subfamily $(13-18,40)$. BMP-2 is currently used as an intervention in spondylodesis, bone defects and osteoporosis (41-43). BMP-2 regulates osteoblast differentiation and later bone formation via a classical TGF- $\beta$ /BMP linear signaling cascade. BMP- 2 is secreted from mesenchymal cells, and then interacts with BMP receptors on the cell membrane, and a subsequent phosphorylation of the Smad transducer occurs. Activated Smad then translocates into the nucleus and BMP and TGF- $\beta$ signals converge to modulate the transcription of numerous osteoblast-specific target genes, namely, the early osteogenic markers ALP, Runx2 and Osx, specifically expressed in developing bones and essential in osteoblast differentiation and bone formation (44-46).

TGF- $\beta 3$ was formerly reported to be an inductive part of the chondrogenic differentiation of progenitor cells $(22,23)$. Exposure of murine induced pluripotent stem cells to TGF- $\beta 3$ in the presence of retinoic acid resulted in bone deposition on ceramic scaffolds implanted in mice (27). Toom et al (47) described an increased level of TGF- $\beta 2$ and TGF- $\beta 3$ during bone formation and remodeling, indicating the implication of TGF- $\beta 3$ in bone formation in heterotopic ossification. Scaffolds infused with BMP- 2 and TGF- $\beta 3$ enhanced bone formation in vivo and improved treatment of the orthotopic defect region, which was consistent with the data from Oest et al (48). A previous study also suggested that craniofacial osteogenesis relied on tight modulation of TGF- $\beta 3$ levels in zebrafish embryos (49). These previous studies indicate TGF- $\beta 3$ may serve as a promoter to accelerate and induce bone formation. However, the osteogenic function of TGF- $\beta 3$ in BMSCs remains to be elucidated.

The present study was the first, to the best of our knowledge, to succeed in delivering BMP- 2 and TGF- $\beta 3$ together into rBMSCs. The RT-PCR and Western blotting results demonstrated that BMP-2/TGF- $\beta 3$ co-transfected rBMSCs expressed markedly elevated quantities of BMP- 2 and TGF- $\beta 3$ proteins, compared with individual gene transfected rBMSCs, indicating that overexpression of TGF- $\beta 3$ ex vivo stimulated the secretion of BMP-2, and vice versa. This was partly consistent 
with a previous study suggesting that the expression of BMP-2 was positively influenced in a time-dependent manner in vivo when pretreated with TGF- $\beta 3$ (28). It was also suggested that TGF- $\beta 3$ elicited bone formation via increasing endogenous BMP-2 levels, and was involved in reprogramming progenitor cells into active secreting osteoblasts (28). In addition, a notable, but as yet unreported, observation is that the addition of TGF- $\beta 3$ increased the osteogenic effect exerted by BMP-2 in vitro, suggesting that Runx-2 and Osx, which are characteristic of early stage bone formation, were markedly upregulated in rBMSCs with BMP-2 and TGF- $\beta 3$ co-expression. Therefore, it was assumed that although TGF- $\beta 3$ did not exert a marked impact on the osteogenic differentiation of BMSCs, it was involved due to increasing the quantity of BMP-2.

TGF- $\beta$ participates in a wide array of processes involved in matrix release and deposition, such as collagen synthesis, including wound healing, angiogenesis, and fibrotic disease. According to Kovacevic et al (50), TGF- $\beta 3$ delivered with a fibrin/heparin composite gel to the healing rotator cuff enthesis resulted in enhanced structural and material properties, and the addition of TGF- $\beta 3$ could expedite healing tendon-bone repair, with an accumulation of osteoconductive calcium-phosphate matrix at the tendon-bone site, associated with new bone formation. This suggested another hypothesis regarding whether TGF- $\beta 3$ promoted bone development by inducing matrix deposition.

The present study conducted a time-dependent measurement of ALP activity, however no investigation into time-dependent Runx-2 and Osx release, or expression levels of BMP-2 and TGF- $\beta 3$ was conducted. The present study also expected to determine whether an interaction existed between BMP-2 and TGF- $\beta 3$, or if TGF- $\beta 3$ collaborated with BMP-2 via a TGF- $\beta$ /BMP signaling pathway. The precise mechanism remains to be elucidated.

In conclusion, the present study, was the first, to the best of our knowledge, to successfully deliver BMP-2 and TGF- $\beta 3$ into BMSCs. The results of the present study demonstrated that combining TGF- $\beta 3$ with BMP- 2 was able to promote the process of bone formation more markedly in vitro, providing a promising clinical strategy in the field of skeletal regeneration and in fracture healing. Future work in the present laboratory would involve research into time-dependent Runx-2 and Osx release, and time-dependent expression levels of BMP-2 and TGF- $\beta 3$. Thus, the mechanism involved in the interplay between BMP-2 and TGF- $\beta 3$, and their reciprocal roles in osteogenesis, may be elucidated.

\section{Acknowledgements}

The present study was supported by the National Natural Science Foundation of China (grant no. 81301570) and the Shandong Natural Science Foundation in China (grant no. 2013ZRCQ018).

\section{References}

1. Kaufmann KB, Büning H, Galy A, Schambach A and Grez M: Gene therapy on the move. EMBO Mol Med 5: 1642-1661, 2013.

2. Wilson JM: Gendicine: The first commercial gene therapy product. Hum Gene Ther 16: 1014-1015, 2005.

3. Cronin M, Stanton R, Francis K and Tangney M: Bacterial vectors for imaging and cancer gene therapy: A review. Cancer Gene Ther 19: 731-740, 2012.
4. Lam P, Khan G, Stripecke R, Hui K, Kasahara N, Peng K and Guinn B: The innovative evolution of cancer gene and cellular therapies. Cancer Gene Ther 20: 141-149, 2013.

5. Jessup M, Greenberg B, Mancini D, Cappola T, Pauly DF, Jaski B, Yaroshinsky A, Zsebo KM, Dittrich H and Hajjar RJ; Calcium Upregulation by Percutaneous Administration of Gene Therapy in Cardiac Disease (CUPID) Investigators: Calcium upregulation by percutaneous administration of gene therapy in cardiac disease (CUPID): A phase 2 trial of intracoronary gene therapy of sarcoplasmic reticulum Ca2+-ATPase in patients with advanced heart failure. Circulation 124: 304-313, 2011.

6. LeWitt PA, Rezai AR, Leehey MA, Ojemann SG, Flaherty AW, Eskandar EN, Kostyk SK, Thomas K, Sarkar A, Siddiqui MS, et al: AAV2-GAD gene therapy for advanced Parkinson's disease: A double-blind, sham-surgery controlled, randomised trial. Lancet Neurol 10: 309-319, 2011.

7. Elsner M, Terbish T, Jörns A, Naujok O, Wedekind D, Hedrich HJ and Lenzen S: Reversal of diabetes through gene therapy of diabetic rats by hepatic insulin expression via lentiviral transduction. Mol Ther 20: 918-926, 2012.

8. Herrera-Carrillo E and Berkhout B: Bone marrow gene therapy for HIV/AIDS. Viruses 7: 3910-3936, 2015.

9. Tian K, Qi M, Wang L, Li Z, Xu J, Li Y, Liu G, Wang B, Huard J and Li G: Two-stage therapeutic utility of ectopically formed bone tissue in skeletal muscle induced by adeno-associated virus containing bone morphogenetic protein-4 gene. J Orthop Surg Res 10: 86, 2015.

10. Tseng SS, Lee MA and Reddi AH: Nonunions and the potential of stem cells in fracture-healing. J Bone Joint Surg Am 1 (90 Suppl): S92-S98, 2008.

11. Wang Y, Zeng B and Li X: Expression of human calcitonin by microencapsulated recombinant myoblasts. Biotechnol Lett 28: 1453-89, 2006.

12. Johnsen SA, Subramaniam M, Katagiri T, Janknecht R and Spelsberg TC: Transcriptional regulation of Smad2 is required for enhancement of TGFbeta/Smad signaling by TGFbeta inducible early gene. J Cell Biochem 87: 233-241, 2002.

13. Kobayashi T, Liu X, Wen FQ, Kohyama T, Shen L, Wang XQ, Hashimoto M, Mao L, Togo S, Kawasaki S, et al: Smad3 mediates TGF-beta1-induced collagen gel contraction by human lung fibroblasts. Biochem Biophys Res Commun 339: 290-295, 2006.

14. Guo L, Zhao RC and $\mathrm{Wu} \mathrm{Y}$ : The role of microRNAs in self-renewal and differentiation of mesenchymal stem cells. Exp Hematol 39: 608-616, 2011.

15. Wang EA, Rosen V, D'Alessandro JS, Bauduy M, Cordes P, Harada T, Israel DI, Hewick RM, Kerns KM and LaPan P: Recombinant human bone morphogenetic protein induces bone formation. Proc Natl Acad Sci USA 87: 2220-2224, 1990.

16. Hay E, Hott M, Graulet AM, Lomri A and Marie PJ: Effects of bone morphogenetic protein-2 on human neonatal calvaria cell differentiation. J Cell Biochem 72: 81-93, 1999.

17. Noshi T, Yoshikawa T, Dohi Y,Ikeuchi M, Horiuchi K, Ichijima K, Sugimura M, Yonemasu K and Ohgushi H: Recombinant human bone morphogenetic protein-2 potentiates the in vivo osteogenic ability of marrow/hydroxyapatite composites. Artif Organs 25: 201-208, 2001.

18. Wilson JM: Adenoviruses as gene-delivery vehicles. N Engl J Med 334: 1185-1187, 1996.

19. LaurencinCT,Attawia MA,LuLQ,Borden MD,LuHH,Gorum WJ and Lieberman JR: Poly (lactide-co-glycolide)/hydroxyapatite delivery of BMP-2-producing cells: A regional gene therapy approach to bone regeneration. Biomaterials 22: 1271-1277, 2001.

20. Huang Z, Ren PG, Ma T, Smith RL and Goodman SB: Modulating osteogenesis of mesenchymal stem cells by modifying growth factor availability. Cytokine 51: 305-10, 2010.

21. Cohen MM Jr: Bone morphogenetic proteins with some comments on fibrodysplasia ossificans progressiva and NOGGIN. Am J Med Genet 109: 87-92, 2002.

22. Guo Q, Liu C, Li J, Zhu C, Yang H and Li B: Gene expression modulation in TGF- $\beta 3$-mediated rabbit bone marrow stem cells using electrospun scaffolds of various stiffness. J Cell Mol Med 19: 1582-1592, 2015.

23. Hara ES, Ono M, Pham HT, Sonoyama W, Kubota S, Kubota S, Takigawa M, Matsumoto T, Young MF, Olsen BR and Kuboki T: Fluocinolone acetonide Is a potent synergistic factor of TGF- $\beta 3$-associated chondrogenesis of bone marrow-derived mesenchymal stem cells for articular surface regeneration. J Bone Miner Res 30: 1585-1596, 2015. 
24. Eduardo K, Hong L and Mao JJ: Inhibition of osteogenic differentiation of human mesenchymal stem cells. Wound Repair Regen 15: 413-9421, 2007.

25. Shi Y and Massagué J: Mechanisms of TGF-beta signaling from cell membrane to the nucleus. Cell 113: 685-700, 2003.

26. Moioli EK, Hong L, Guardado J, Clark PA and Mao JJ: Sustained Release of TGFbeta3 from PLGA microspheres and its effect on early osteogenic differentiation of human mesenchymal stem cells tissue eng 12: 537-546, 2006.

27. Li F and Niyibizi C: Cells derived from murine induced pluripotent stem cells (iPSC) by treatment with members of TGF-beta family give rise to osteoblasts differentiation and form bone in vivo. BMC Cell Biol 13: 35, 2012.

28. Klar RM, Duarte R, Dix-Peek T and Ripamonti U: The induction of bone formation by the recombinant human transforming growth factor- $\beta 3$. Biomaterials 35: 2773-2788, 2014.

29. Haschtmann D, Ferguson SJ and Stoyanov JV: BMP-2 and TGF- $\beta 3$ do not prevent spontaneous degeneration in rabbit disc explants but induce ossification of the annulus fibrosus. Eur Spine J 21: 1724-1733, 2012.

30. He T, Wang Y, Xiang $\mathrm{J}$ and Zhang $\mathrm{H}$ : In vivo tracking of novel SPIO-Molday ION rhodamine-B ${ }^{\mathrm{TM}}$-labeled human bone marrow-derived mesenchymal stem cells after lentivirus-mediated COX-2 silencing: A preliminary study. Curr Gene Ther 14: 136-145, 2014.

31. Alhadlaq A and Mao JJ: Mesenchymal stem cells: Isolation and therapeutics. Stem Cells Dev 13: 436-448, 2004.

32. Parekkadan B and Milwid JM: Mesenchymal stem cells as therapeutics. Annu Rev Biomed Eng 12: 87-1174, 2010.

33. Bianco P, Riminucci M, Gronthos S and Robey PG: Bone marrow stromal stem cells: Nature, biology, and potential applications. Stem Cells 19: 180-192, 2001.

34. Derubeis AR and Cancedda R: Bone marrow stromal cells (BMSCs) in bone engineering: Limitations and recent advances. Ann. Biomed. Eng 32: 160-165, 2004.

35. Hu H, Hilton MJ, Tu X, Yu K, Ornitz DM and Long F: Sequential roles of hedgehog and wnt signaling in osteoblast development. Development 132: 49-60, 2005.

36. Forriol F and Shapiro F: Bone development: Interaction of molecular components and biophysical forces. Clin Orthop Relat Res 432: 14-33, 2005.

37. Gordon KJ and Blobe GC: Role of transforming growth factor-beta superfamily signaling pathways in human disease. Biochim Biophys Acta 1782: 197-228, 2008

38. Herpin A, Lelong $C$ and Favrel P: Transforming growth factor-beta-related proteins: An ancestral and widespread superfamily of cytokines in metazoans. Dev Comp Immunol 28: $461-485,2004$
39. Assoian RK, Komoriya A, Meyers CA, Miller DM and Sporn MB: Transforming growth factor-beta in human platelets. Identification of a major storage site, purification and characterization. J Biol Chem 258: 7155-7160, 1983.

40. Ripamonti U: Soluble osteogenic molecular signals and the induction of bone formation. Biomaterials 27: 807-822, 2006.

41. Carreira AC, Lojudice FH, Halcsik E, Navarro RD, Sogayar MC and Granjeiro JM: Bone morphogenetic proteins: Facts, challenges, and future perspectives. J Dent Res 93: 335-345, 2014.

42. Burkus JK, Sandhu HS, Gornet MF and Longley MC: Use of rhBMP-2 in combination with structural cortical allografts: Clinical and radiographic outcomes in anterior lumbar spinal surgery. J Bone Joint Surg Am 87: 1205-1212, 2005.

43. Meisel HJ, Schnöring M, Hohaus C, Minkus Y, Beier A, Ganey T and Mansmann U: Posterior lumbar interbody fusion using rhBMP-2. Eur Spine J 17: 1735-1744, 2008.

44. Rahman MS, Akhtar N, Jamil HM, Banik RS and Asaduzzaman SM: TGF-b/BMP signaling and other molecular events: Regulation of osteoblastogenesis and bone formation. Bone Research 3: 15005, 2015.

45. ten Dijke P, Miyazono K and Heldin CH: Signaling inputs converge on nuclear effectors in TGF-beta signaling. Trends Biochem Sci 25: 64-70, 2000.

46. Kusanagi K, Inoue H, Ishidou Y, Mishima HK, Kawabata M and Miyazono K: Characterization of a bone morphogenetic protein-responsive Smad-binding element. Mol Biol Cell 11: $555-565,2000$

47. Toom A, Arend A, Gunnarsson D, Ulfsparre R, Suutre S, Haviko T and Selstam G: Bone formation zones in heterotopic ossifications: Histologic findings and increased expression of bonemorphogenetic protein 2 and transforming growth factors beta 2 and beta3. Calcif Tissue Int 80: 259-267, 2007.

48. Oest ME, Dupont KM, Kong HJ, Mooney DJ and Guldberg RE: Quantitative assessment of scaffold and growth factor-mediated repair of critically sized bone defects. J Orthop Res 25: 941-950, 2007.

49. Cheah FS, Winkler C, Jabs EW and Chong SS: Tgfbeta3 regulation of chondrogenesis and osteogenesis in zebrafish is mediated through formation and survival of a subpopulation of the cranial neural crest. Mech Dev 127: 329-344, 2010.

50. Kovacevic D, Fox JA, Bedi A, Ying L, Deng XH, Warren RF and Rodeo AS: Calcium-phosphate matrix with or without TGF- $\beta 3$ improves tendon-bone healing after rotator cuff repair. Am J Sports Med 39: 811-819, 2011. 